\title{
Convexity Properties for Certain Classes of Analytic Functions Associated with an Integral Operator
}

\author{
Ben Wongsaijai and Nattakorn Sukantamala \\ Department of Mathematics, Faculty of Science, Chiang Mai University, Chiang Mai 50200, Thailand \\ Correspondence should be addressed to Nattakorn Sukantamala; gaia2556@gmail.com
}

Received 30 May 2014; Revised 4 September 2014; Accepted 4 September 2014; Published 14 October 2014

Academic Editor: Valery Serov

Copyright (C) 2014 B. Wongsaijai and N. Sukantamala. This is an open access article distributed under the Creative Commons Attribution License, which permits unrestricted use, distribution, and reproduction in any medium, provided the original work is properly cited.

\begin{abstract}
We introduce a new form of generalized integral operator defined on the class of analytic functions $\mathscr{A}_{0}$. By making use of this novel integral operator, we give the convexity of other integral operators. We also briefly indicate the relevant connections of our presented results to the formerly reported results. Furthermore, other interesting properties are also discussed.
\end{abstract}

\section{Introduction}

In the field of geometric function theory, the class of univalent functions $[1,2]$ has been mainly studied. There are many distinguished geometric properties that played important role in the theory of univalent functions, such as starlikeness, convexity, and close-to-convexity (see, e.g., [3-5]). One of the generalizations of univalent functions is the theory of multivalent functions or $p$-valent functions. Also, the geometric properties for the subclasses of $p$-valent functions are investigated by many authors (see, e.g., [6-9]).

Let $\mathbb{D}=\{z \in \mathbb{C}:|z|<1\}$ be an open unit disc in the complex plane. For a positive integer $p, \mathscr{A}_{p}$ denotes the class of $p$-valent functions of the following form:

$$
f(z)=z^{p}+\sum_{j=1}^{\infty} a_{p+j} z^{p+j}
$$

which is analytic in $\mathbb{D}$. In particular, we set $\mathscr{A}_{1} \equiv \mathscr{A}$. Furthermore, let $\mathscr{A}_{0}$ be the class of analytic functions $f$ in $\mathbb{D}$ of the following form:

$$
f(z)=1+\sum_{j=1}^{\infty} b_{j} z^{j}
$$

A function $f \in \mathscr{A}_{p}$ is said to be $p$-valently starlike of order $\gamma(0 \leq \gamma<p)$ in $\mathbb{D}$ if $f$ satisfies

$$
\operatorname{Re}\left\{\frac{z f^{\prime}(z)}{f(z)}\right\}>\gamma, \quad z \in \mathbb{D} \text {. }
$$

We denote this class by $\mathcal{S}_{p}^{*}(\gamma)$ which was introduced by Patil and Thakare [10]. In particular, we set $\mathcal{S}_{p}^{*}(0) \equiv \mathcal{S}_{p}^{*}$ for a class of $p$-valent starlike functions in $\mathbb{D}$. Denoted by $\mathcal{S}^{*}(p, \gamma)$, the subclass of $\mathcal{S}_{p}^{*}(\gamma)$ consists of functions $f \in \mathscr{A}_{p}$ for which

$$
\left|\frac{z f^{\prime}(z)}{f(z)}-p\right|<p-\gamma, \quad z \in \mathbb{D} .
$$

On the other hand, a function $f \in \mathscr{A}_{p}$ is said to be $p$-valently convex of order $\gamma(0 \leq \gamma<p)$ in $\mathbb{D}$ if $f$ satisfies

$$
\operatorname{Re}\left\{1+\frac{z f^{\prime \prime}(z)}{f^{\prime}(z)}\right\}>\gamma, \quad z \in \mathbb{D} .
$$

We denote this class by $\mathscr{K}_{p}(\gamma)$ which was introduced by Owa [11]. In particular, we set $\mathscr{K}_{p}(0) \equiv \mathscr{K}_{p}$ for a class of $p$ valent convex functions in $\mathbb{D}$. Analogously, we denote that the subclass $\mathscr{K}(p, \gamma)$ of $\mathscr{K}_{p}(\gamma)$ consists of functions $f \in \mathscr{A}_{p}$ for which

$$
\left|1+\frac{z f^{\prime \prime}(z)}{f^{\prime}(z)}-p\right|<p-\gamma, \quad z \in \mathbb{D} .
$$


By using the Alexander-type criterion, it follows that

$$
f(z) \in \mathscr{K}_{p}(\gamma) \Longleftrightarrow \frac{z f^{\prime}(z)}{p} \in \mathcal{S}_{p}^{*}(\gamma) .
$$

The statement is also true if we replace $\mathcal{S}_{p}^{*}(\gamma)$ and $\mathscr{K}_{p}(\gamma)$ by $\mathcal{S}^{*}(p, \gamma)$ and $\mathscr{K}(p, \gamma)$, respectively. Moreover, we note that

$$
\mathcal{S}^{*}(p, \gamma) \subset \mathcal{S}_{p}^{*}(\gamma) \subset \mathcal{S}_{p}^{*}, \quad \mathscr{K}(p, \gamma) \subset \mathscr{K}_{p}(\gamma) \subset \mathscr{K}_{p} .
$$

A function $f \in \mathcal{U} \mathcal{S}_{p}(\delta, \gamma)$ is said to be $\delta$-uniformly $p$ valent starlike of order $\gamma(-1 \leq \gamma<p, \delta \geq 0)$ in $\mathbb{D}$ if $f$ satisfies

$$
\operatorname{Re}\left\{\frac{z f^{\prime}(z)}{f(z)}\right\}>\delta\left|\frac{z f^{\prime}(z)}{f(z)}-p\right|+\gamma, \quad z \in \mathbb{D} .
$$

Furthermore, a function $f \in \mathcal{U} \mathscr{K}_{p}(\delta, \gamma)$ is said to be $\delta$ uniformly $p$-valent starlike of order $\gamma(-1 \leq \gamma<p, \delta \geq 0)$ in $\mathbb{D}$ if $f$ satisfies

$$
\operatorname{Re}\left\{1+\frac{z f^{\prime \prime}(z)}{f^{\prime}(z)}\right\}>\delta\left|1+\frac{z f^{\prime \prime}(z)}{f^{\prime}(z)}-p\right|+\gamma, \quad z \in \mathbb{D} .
$$

Both $\mathcal{U} \mathcal{S}_{p}(\delta, \gamma)$ and $\mathcal{U} \mathscr{K}_{p}(\delta, \gamma)$ are comprehensive classes of analytic functions that include various classes of analytic univalent functions as well as some very well-known ones. For example, in the case $p=1$, we have $\mathcal{U} \mathcal{S}_{1}(\delta, \gamma) \equiv$ $\mathcal{U} \delta(\delta, \gamma)$ and $\mathscr{U} \mathscr{K}_{1}(\delta, \gamma) \equiv \mathscr{U} \mathscr{K}(\delta, \gamma)$ which are introduced by Bharati et al. [12]. For $\gamma=0, \mathscr{U} \mathscr{K}_{1}(\delta, 0) \equiv \mathscr{U} \mathscr{K}(\delta)$ is the class of $\delta$-uniformly convex function [13]. In the special case, $p=\delta=1$ and $\gamma=0$, the class $\mathcal{U} \mathcal{S}_{1}(1,0) \equiv \mathscr{U} \mathcal{S}$ of uniformly starlike functions and $\mathscr{U} \mathscr{K}_{1}(1,0) \equiv \mathcal{U} \mathscr{K}$ of uniformly convex functions were introduced by Goodman $[14,15]$. Using the Alexander type relation, statement (7) holds for the $\mathcal{U} \mathscr{K}_{p}(\beta, \gamma)$ and $\mathcal{U} \mathcal{S}_{p}(\beta, \gamma)$; that is,

$$
f(z) \in \mathcal{U} \mathscr{K}_{p}(\beta, \gamma) \Longleftrightarrow \frac{z f^{\prime}(z)}{p} \in \mathcal{U} \delta_{p}(\beta, \gamma) .
$$

Many researchers have studied the geometric properties of integral operators. The common investigation is finding sufficient conditions of integral operators in order to transform analytic functions into classes with each of those mentioned properties. The well-known integral transformation defining a subclass of univalent functions was introduced by Alexander in [16]. It is of the following form:

$$
F[f](z)=\int_{0}^{z} \frac{f(t)}{t} d t
$$

In [17], Kim and Merkes extended the integral operator (12) by introducing a complex parameter $\alpha$ as

$$
F^{\alpha}[f](z)=\int_{0}^{z}\left(\frac{f(t)}{t}\right)^{\alpha} d t .
$$

Another object of investigation for the studies of the integral operator by Pfaltzgraff [18] is $G^{\alpha}$ defined by

$$
G^{\alpha}[f](z)=\int_{0}^{z}\left(f^{\prime}(t)\right)^{\alpha} d t .
$$

Until now, the various generalized form of the integral operators $F^{\alpha}$ in (13) and $G^{\alpha}$ in (14) has been investigated. However, Breaz and Stanciu [19] introduced and studied the more general form of integral operator $J_{n}^{\alpha, \mu}: \mathscr{A}^{n} \times \mathscr{A}^{n} \rightarrow \mathscr{A}$, which is

$$
J_{n}^{\alpha, \mu}[f, g](z)=\int_{0}^{z} \prod_{k=1}^{n}\left(\frac{f_{k}(t)}{t}\right)^{\alpha_{k}}\left(g_{k}^{\prime}(t)\right)^{\mu_{k}} d t .
$$

By setting appropriate values for the parameters $n, \alpha$, and $\mu$, integral operators that have been previously introduced can be obtained. In particular, if $\mu_{k}=0$, then the integral operator $J_{n}^{\alpha, 0}$ becomes the integral operator $F_{n}^{\alpha}$ introduced by D. Breaz and N. Breaz [20]. Also, when $\alpha_{k}=0$, the integral operator $J_{n}^{0, \mu}$ is exactly the integral operator $G_{n}^{\alpha}$ defined by Breaz et al. [21]. The specialized form of $F_{n}^{\alpha}$ and $G_{n}^{\alpha}$ involving the Bessel functions was introduced and studied in [22-24]. In addition, the specific case $n=1$ for $J_{n}^{\alpha, \mu}$ in (15), $J_{1}^{\alpha, \mu}=J^{\alpha, \mu}$ was investigated by Pescar in [25]. The univalence and their properties of the integral operators are reported in [26-30].

In [31], Bulut developed the integral operator $I_{n}^{\alpha, \mu}: \mathscr{A}_{p}^{n} \times$ $\mathscr{A}_{p}^{n} \rightarrow \mathscr{A}_{p}$ which extends the class of analytic functions $\mathscr{A}$ to the class of $p$-valent functions $\mathscr{A}_{p}$; that is,

$$
I_{p, n}^{\alpha, \mu}[f, g](z)=\int_{0}^{z} p t^{p-1} \prod_{k=1}^{n}\left(\frac{f_{k}(t)}{t^{p}}\right)^{\alpha_{k}}\left(\frac{g_{k}^{\prime}(t)}{p t^{p-1}}\right)^{\mu_{k}} d t .
$$

By setting $\mu_{k}=0$ and $\alpha_{k}=0$, we obtain the integral operators $I_{p, n}^{\alpha, 0}=F_{p, n}^{\alpha}$ and $I_{p, n}^{0, \mu}=G_{p, n}^{\mu}$, respectively, which were introduced by Frasin [32]. Also, some properties of these integral operators have been studied in [32-34].

Recently, many authors modified integral operators associated with differential operator such as Salagean operator [35], Ruscheweyh operator [36], Al-Oboudi operator [37], and Carlson-Shaffer operator [38]. In [39], Frasin investigated one of the generalized integral operators by using the Hadamard product to demonstrate most of the previously defined integral operators. Frasin [39] defined the integral operator $H_{n}: \mathscr{A}^{n} \times \mathscr{A}^{n} \rightarrow \mathscr{A}$ by

$$
H_{n}[f, g](z)=\int_{0}^{z} \prod_{k=1}^{n}\left(\frac{\left(f_{k} * g_{k}\right)(t)}{t}\right)^{\alpha_{k}} d t
$$

where $(f * g)(z) / z \neq 0, z \in \mathbb{D}$ and the Hadamard product is defined by

$$
(f * g)(z) \equiv z+\sum_{j=2}^{\infty} a_{j} b_{j} z^{j}, \quad z \in \mathbb{D},
$$

where $f(z)=z+\sum_{j=2}^{\infty} a_{j} z^{j}$ and $g(z)=z+\sum_{j=2}^{\infty} b_{j} z^{j}$. It was reported that, for appropriate functions $g_{k} \in \mathscr{A}$, the integral operator $H_{n}$ generalizes many integral operators introduced and studied by several authors [40-44]. Moreover, the integral operator $H_{n}$ is generalized integral operators of those in (12)-(15). In a similar idea, $H_{n}$ can be extended to more generalized on $\mathscr{A}_{p}^{n} \times \mathscr{A}_{p}^{n}$ to $\mathscr{A}_{p}$ by

$$
H_{p, n}[f, g](z)=\int_{0}^{z} p t^{p-1} \prod_{k=1}^{n}\left(\frac{\left(f_{k} * g_{k}\right)(t)}{t^{p}}\right)^{\alpha_{k}} d t
$$


where $(f * g)(z) / z^{p} \neq 0, f(z)=z^{p}+\sum_{j=1}^{\infty} a_{p+j} z^{p+j}$, and $g(z)=z^{p}+\sum_{j=1}^{\infty} b_{p+j} z^{p+j}$, and the Hadamard product is defined by

$$
(f * g)(z) \equiv z^{p}+\sum_{j=1}^{\infty} a_{p+j} b_{p+j} z^{p+j}, \quad z \in \mathbb{D} .
$$

Certainly, the integral operator $H_{p, n}$ generalizes many operators when we choose suitable functions $g_{k} \in \mathscr{A}_{p}$ (see, e.g., [45-47]).

In fact, we can write the Hadamard product in the form $(f * g)(z)=z^{p} h(z)$ where $h$ is analytic in $\mathbb{D}$ and $h(0)=1$. Indeed,

$$
h(z)=1+\sum_{j=1}^{\infty} a_{p+j} b_{p+j} z^{j}, \quad z \in \mathbb{D},
$$

which usually appears in most of integral operators and always belongs to the class $\mathscr{A}_{0}$. That is why we are interested in replacing the term $\left(f_{k} * g_{k}\right)(z) / z^{p}$ in the integral operator (19) with general function in $\mathscr{A}_{0}$. Additionally, replacing the term $z^{p-1}$ with a function $\phi(z) \in \mathscr{A}_{p-1}$ yields the integral operator, which is still contained in $\mathscr{A}_{p}$.

We now define the following general integral operator $\mathscr{J}_{\phi}^{\alpha}: \mathscr{A}_{0}^{n} \rightarrow \mathscr{A}_{p}$, for $p \geq 1, \phi(z) \in \mathscr{A}_{p-1}$, and $\alpha=$ $\left(\alpha_{1}, \alpha_{2}, \ldots, \alpha_{n}\right) \in \mathbb{R}_{+}^{n}$ by

$$
\mathcal{F}_{\phi}^{\alpha}[h](z)=\int_{0}^{z} p \phi(t) \prod_{k=1}^{n}\left(h_{k}(t)\right)^{\alpha_{k}} d t,
$$

where $h_{k} \in \mathscr{A}_{0}$ for all $k=1,2, \ldots, n$.

The main purpose of the paper is to investigate the sufficient conditions on convexity of the integral operator $\mathcal{F}_{\phi}^{\alpha}[h]$ on classes $\mathcal{S}_{p}^{*}(\gamma), \mathcal{S}^{*}(p, \gamma)$, and $\mathcal{U} \mathcal{S}(\delta, \gamma)$ of analytic functions. Our main results will be applied to reinstate the results of former researches with related integral operators.

\section{Main Results}

In this section, we investigate sufficient conditions for the convexity of the integral operator $\mathscr{F}_{\phi}^{\alpha}[h]$ which is defined by (22). For the convenience, we introduce the transformation operator $\mathscr{T}_{p}: \mathscr{A}_{q} \rightarrow \mathscr{A}_{p+q}$ by

$$
\mathscr{T}_{p}(f)(z)=z^{p} f(z), \quad z \in \mathbb{D},
$$

where $f \in \mathscr{A}_{q}$ and $q$ is a nonnegative integer. In particular, we set $\mathscr{T}_{1}=\mathscr{T}$.

We now prove a general property which guarantees the convexity of the proposed integral operator on the class $\mathcal{S}_{p}^{*}(\gamma)$

Theorem 1. Let $0 \leq \lambda, \gamma_{k}<p, \mathscr{T}(\phi) \in \mathcal{S}_{p}^{*}(\lambda)$, and $\mathscr{T}_{p}\left(h_{k}\right) \in$ $\mathcal{S}_{p}^{*}\left(\gamma_{k}\right)$ for $k=1,2, \ldots, n$. If $\alpha=\left(\alpha_{1}, \alpha_{2}, \ldots, \alpha_{n}\right) \in \mathbb{R}_{+}^{n}$ satisfies

$$
\sum_{k=1}^{n} \alpha_{k}\left(p-\gamma_{k}\right) \leq \lambda
$$

then the integral operator $\mathscr{F}_{\phi}^{\alpha}[h]$ defined by (22) is p-valently convex of order $\lambda-\sum_{k=1}^{n} \alpha_{k}\left(p-\gamma_{k}\right)$.

Proof. From the definition of integral opeartor in (22), we observe that $\mathscr{I}_{\phi}^{\alpha}[h] \in \mathscr{A}_{p}$. By calculating the first derivative of $\mathcal{F}_{\phi}^{\alpha}[h]$, we obtain

$$
\left(\mathscr{F}_{\phi}^{\alpha}[h]\right)^{\prime}(z)=p \phi(z) \prod_{k=1}^{n}\left(h_{k}(z)\right)^{\alpha_{k}} .
$$

Differentiating on both sides of (25) logarithmically and multiplying by $z$ give

$$
\frac{z\left(\mathscr{F}_{\phi}^{\alpha}[h]\right)^{\prime \prime}(z)}{\left(\mathscr{F}_{\phi}^{\alpha}[h]\right)^{\prime}(z)}=\frac{z \phi^{\prime}(z)}{\phi(z)}+\sum_{k=1}^{n} \alpha_{k} \frac{z h_{k}^{\prime}(z)}{h_{k}(z)} .
$$

Since $\mathscr{T}(\phi) \in \mathcal{S}_{p}^{*}(\lambda)$, it follows that

$$
\begin{aligned}
\operatorname{Re}\left\{\frac{z \mathscr{T}(\phi(z))^{\prime}(z)}{\mathscr{T}(\phi)(z)}\right\} & =\operatorname{Re}\left\{\frac{z(z \phi(z))^{\prime}(z)}{z \phi(z)}\right\} \\
& =\operatorname{Re}\left\{1+\frac{z \phi^{\prime}(z)}{\phi(z)}\right\}>\lambda .
\end{aligned}
$$

Also, since $\mathscr{T}_{p}\left(h_{k}\right) \in \mathcal{S}_{p}^{*}\left(\gamma_{k}\right)$, we have

$$
\begin{aligned}
\operatorname{Re}\left\{\frac{z\left(\mathscr{T}_{p}\left(h_{k}\right)\right)^{\prime}(z)}{\mathscr{T}_{p}\left(h_{k}\right)(z)}\right\} & =\operatorname{Re}\left\{\frac{z\left(z^{p} h_{k}\right)^{\prime}(z)}{z^{p} h_{k}(z)}\right\} \\
& =\operatorname{Re}\left\{p+\frac{z h_{k}^{\prime}(z)}{h_{k}(z)}\right\}>\gamma_{k} .
\end{aligned}
$$

From (27) and (28), by taking the real part of (26), we obtain

$$
\begin{aligned}
\operatorname{Re}\left\{1+\frac{z\left(\mathscr{F}_{\phi}^{\alpha}[h]\right)^{\prime \prime}(z)}{\left(\mathcal{F}_{\phi}^{\alpha}[h]\right)^{\prime}(z)}\right\} \\
=\operatorname{Re}\left\{1+\frac{z \phi^{\prime}(z)}{\phi(z)}\right\}+\sum_{k=1}^{n} \alpha_{k} \operatorname{Re}\left\{\frac{z h_{k}^{\prime}(z)}{h_{k}(z)}\right\} \\
>\lambda+\sum_{k=1}^{n} \alpha_{k} \operatorname{Re}\left\{p+\frac{z h_{k}^{\prime}(z)}{h_{k}(z)}\right\}-p \sum_{k=1}^{n} \alpha_{k} \\
>\lambda-\sum_{k=1}^{n} \alpha_{k}\left(p-\gamma_{k}\right) .
\end{aligned}
$$

Therefore, $\mathscr{J}_{\phi}^{\alpha}[h]$ is $p$-valently convex of order $\lambda-\sum_{k=1}^{n} \alpha_{k}(p-$ $\left.\gamma_{k}\right)$.

We note that by suitable functions $\phi \in \mathscr{A}_{p-1}, h_{k} \in \mathscr{A}_{0}$, and $\left(\alpha_{1}, \alpha_{2}, \ldots, \alpha_{n}\right) \in \mathbb{R}_{+}^{n}$ in Theorem 1, we obtain the earlier result. For example, if $p=1$ and $\phi(z)=1$, we obtain Theorem 2.1 in [44] and Theorems 2.1 and 2.2 in [48]. If $p \geq 1$ and $\phi(z)=z^{p-1}$, by using the Alexander type relation (7), Theorem 1 in [19] is obtained. 
Using the same method and technique as that in Theorem 1 with the nonnegativity of modulus of complex numbers, we are led easily to Theorem 2 . The proof is omitted.

Theorem 2. Let $\mathscr{T}(\phi) \in \mathscr{U} \mathcal{S}_{p}(\delta, \lambda)$ and $\mathscr{T}_{p}\left(h_{k}\right) \in$ $\mathcal{U} \mathcal{S}_{p}\left(\delta_{k}, \gamma_{k}\right)$ for $k=1,2, \ldots, n$. If $\alpha=\left(\alpha_{1}, \alpha_{2}, \ldots, \alpha_{n}\right) \in \mathbb{R}_{+}^{n}$ satisfies

$$
\sum_{k=1}^{n} \alpha_{k}\left(p-\gamma_{k}\right) \leq \lambda
$$

then the integral operator $\mathscr{F}_{\phi}^{\alpha}[h]$ defined by (22) is p-valently convex of order $\lambda-\sum_{k=1}^{n} \alpha_{k}\left(p-\gamma_{k}\right)$.

Theorem 2 generalizes many results proposed by several authors. For $p=1$ and $\phi(z)=1$, we obtain Theorem 2.1 in [26] and Theorem 2 in [49]. For $p \geq 1$ and $\phi(z)=z^{p-1}$ with the Alexander type relation (11), we obtain Theorem 2 in [46] and Theorem 2.1 in [31]. Also, Theorems 2.1 and 3.1 in [33] are obtained.

The following is a result on the transformation property of $\mathcal{F}_{\phi}^{\alpha}[h]$ on the class $\mathcal{S}^{*}(p, \gamma)$.

Theorem 3. Let $\mathscr{T}(\phi) \in \mathcal{S}^{*}(p, \lambda)$ and $\mathscr{T}_{p}\left(h_{k}\right) \in \mathcal{S}^{*}\left(p, \gamma_{k}\right)$ for $k=1,2, \ldots, n$. If $\alpha=\left(\alpha_{1}, \alpha_{2}, \ldots, \alpha_{n}\right) \in \mathbb{R}_{+}^{n}$ satisfies

$$
\sum_{k=1}^{n} \alpha_{k}\left(p-\gamma_{k}\right) \leq \lambda
$$

then the integral operator $\mathscr{F}_{\phi}^{\alpha}[h]$ defined by (22) is in the class $\mathscr{K}\left(p, \lambda-\sum_{k=1}^{n} \alpha_{k}\left(p-\gamma_{k}\right)\right)$. Furthermore, $\mathscr{J}_{\phi}^{\alpha}[h]$ is p-valently convex of order $\lambda-\sum_{k=1}^{n} \alpha_{k}\left(p-\gamma_{k}\right)$.

Proof. From (26), we obtain

$$
\begin{aligned}
\left|1+\frac{z\left(\mathscr{I}_{\phi}^{\alpha}[h]\right)^{\prime \prime}(z)}{\left(\mathscr{J}_{\phi}^{\alpha}[h]\right)^{\prime}(z)}-p\right| \leq & \left|1+\frac{z \phi^{\prime}(z)}{\phi(z)}-p\right| \\
& +\sum_{k=1}^{n} \alpha_{k}\left|\frac{z h_{k}^{\prime}(z)}{h_{k}(z)}\right| .
\end{aligned}
$$

Since $\mathscr{T}(\phi) \in \mathcal{S}^{*}(p, \lambda)$, it follows that

$$
\begin{aligned}
\left|\frac{z(\mathscr{T}(\phi))^{\prime}(z)}{\mathscr{T}(\phi)(z)}-p\right| & =\left|\frac{z(z \phi)^{\prime}(z)}{z \phi(z)}-p\right| \\
& =\left|1+\frac{z \phi^{\prime}(z)}{\phi(z)}-p\right|<p-\lambda .
\end{aligned}
$$

Also, since $\mathscr{T}_{p}\left(h_{k}\right) \in \mathcal{S}^{*}\left(p, \gamma_{k}\right)$, we have

$$
\begin{aligned}
\left|\frac{z\left(\mathscr{T}_{p}\left(h_{k}\right)\right)^{\prime}(z)}{\mathscr{T}_{p}\left(h_{k}\right)(z)}-p\right| & =\left|\frac{z\left(z^{p} h_{k}\right)^{\prime}(z)}{z^{p} h_{k}(z)}-p\right| \\
& =\left|\frac{z h_{k}^{\prime}(z)}{h_{k}(z)}\right|<p-\gamma_{k} .
\end{aligned}
$$

By substitution of (33) and (34) into (32), we get

$$
\left|1+\frac{z\left(\mathscr{F}_{\phi}^{\alpha}[h]\right)^{\prime \prime}(z)}{\left(\mathscr{F}_{\phi}^{\alpha}[h]\right)^{\prime}(z)}-p\right| \leq p-\lambda+\sum_{k=1}^{n} \alpha_{k}\left(p-\gamma_{k}\right) .
$$

Therefore, $\mathscr{J}_{\phi}^{\alpha}[h] \in \mathscr{K}\left(p, \gamma-\sum_{k=1}^{n} \alpha_{k}\left(p-\beta_{k}\right)\right)$. It follows that $\mathscr{J}_{\phi}^{\alpha}[h]$ is $p$-valently convex of order $\lambda-\sum_{k=1}^{n} \alpha_{k}\left(p-\gamma_{k}\right)$.

Remark 4. The parameter $\alpha=\left(\alpha_{1}, \alpha_{2}, \ldots, \alpha_{n}\right)$ in Theorem 3 can be extended to the complex number and assumption (31) becomes

$$
\sum_{k=1}^{n}\left|\alpha_{k}\right|\left(p-\gamma_{k}\right) \leq \lambda
$$

That is, Theorem 3 can be applied to the integral operator $\mathscr{J}_{\phi}^{\alpha}[h]$ in case $\alpha=\left(\alpha_{1}, \alpha_{2}, \ldots, \alpha_{n}\right) \in \mathbb{C}^{n}$.

Over the past few decades, there are many studies on the sufficient conditions that make the integral operators univalent. In fact, the class of convex functions is a subclass of the class of all univalent functions in $\mathbb{D}$. Thus, it is interesting to observe that many results on the univalence property of integral operators follow the convexity property according to main results, especially Theorem 3 or Remark 4 .

We now consider the integral operator $H_{n}: \mathscr{A}^{n} \times \mathscr{A}^{n} \rightarrow$ $\mathscr{A}$ defined in (17). In order to obtain the convexity of the integral operator $H_{n}$ by Theorem 3 or Remark 4 , we set $\phi(z)=$ $1, p=1, \gamma_{k}=0, \alpha=\left(\alpha_{1}, \alpha_{2}, \ldots, \alpha_{n}\right) \in \mathbb{C}^{n}$, and

$$
h_{k}(z)=\frac{\left(f_{k} * g_{k}\right)(z)}{z} \in \mathscr{A}_{0}, \quad k=1,2, \ldots, n,
$$

where $f_{k}, g_{k} \in \mathscr{A}$. Other than that, the univalent property of $H_{n}$ is also obtained. This implies Theorem 3.1 of Frasin in [39]. Moreover, Frasin [39] noticed that for suitable functions $g_{k} \in \mathscr{A}$, the integral operator $H_{n}$ generalizes many operators introduced by several authors, for instance, Theorem 1 in [20], Theorem 2.1 in [41], Theorem 2.3 in [42], and Theorem 2.3 in [43]. It is noteworthy to say that, under same assumptions, the former researches obtain only the univalence, while we obtain the stronger result, which is the convexity.

Our results can be used to explain the convexity of the other integral operators that are related to the Hadamad product as described next.

Remark 5. For $p \geq 1$, we set $\phi(z)=z^{p-1}$ and take

$$
h_{k}(z)=\frac{\left(f_{k} * g_{k}\right)(z)}{z^{p}} \in \mathscr{A}_{0}, \quad k=1,2, \ldots, n,
$$

where $f_{k}, g_{k} \in \mathscr{A}_{p}$. Then, we can apply the main results to discuss the convexity of the integral operator $H_{p, n}$ defined by (19).

Remark 6. The main results are also applicable to the integral operator $G_{p, n}: \mathscr{A}_{p}^{n} \times \mathscr{A}_{p}^{n} \rightarrow \mathscr{A}_{p}$ of the following form:

$$
G_{p, n}[f, g](z)=\int_{0}^{z} p t^{p-1} \prod_{k=1}^{n}\left(\frac{\left(f_{k} * g_{k}\right)^{\prime}(t)}{p t^{p-1}}\right)^{\alpha_{k}} d t,
$$


where $f_{k}, g_{k} \in \mathscr{A}_{p}$. In order to apply the main results, by the Alexander-type criterion, we note that

$$
\begin{aligned}
\mathscr{T}_{p}\left(\frac{\left(f_{k} * g_{k}\right)^{\prime}(z)}{p z^{p-1}}\right) \in \mathcal{S}_{p}^{*}(\gamma) \\
\Longleftrightarrow \frac{z\left(f_{k} * g_{k}\right)^{\prime}(z)}{p} \in \mathcal{S}_{p}^{*}(\gamma) \\
\Longleftrightarrow\left(f_{k} * g_{k}\right)(z) \in \mathscr{K}_{p}(\gamma) .
\end{aligned}
$$

The above statement also holds for the pairs of classes $\mathcal{U} \mathcal{S}_{p}(\beta, \gamma)-\mathcal{U} \mathscr{K}_{p}(\beta, \gamma)$ and $\mathcal{S}(p, \gamma)-\mathscr{K}(p, \gamma)$.

Remark 7. For suitable functions $g_{k} \in \mathscr{A}_{p}$, by Remarks 5 and 6 , we obtain new results for the convexity of other integral operators, for example, $\mathscr{F}_{p, \delta, l}^{n, m}$ and $\mathscr{G}_{p, \delta, l}^{n, m}$ in [45], $\mathscr{F}_{p, n, l, \delta}$ and $\mathscr{G}_{p, n, l, \delta}$ in [46], $\mathscr{F}_{p, m, l, \mu}$ and $\mathscr{G}_{p, m, l, \mu}$ in [50], and so forth.

\section{Conflict of Interests}

The authors declare that there is no conflict of interests regarding the publication of this paper.

\section{Acknowledgments}

The authors sincerely thank the reviewers for their valuable suggestions and useful comments that improved the presentation of the paper. This research was supported by Science Achievement Scholarship of Thailand (SAST) and Department of Mathematics, Faculty of Science, Chiang Mai University.

\section{References}

[1] C. Pommerenke, Univalent Functions, Vandemhoeck and Ruprecht, Göttingen, Germany, 1975.

[2] A. W. Goodman, Univalent Functions, vol. 1-2, Mariner, Tampa, Fla, USA, 1983.

[3] M. I. S. Robertson, "On the theory of univalent functions," Annals of Mathematics, vol. 37, no. 2, pp. 374-408, 1936.

[4] A. Schild, "On a class of univalent, star shaped mappings," Proceedings of the American Mathematical Society, vol. 9, pp. 751-757, 1958.

[5] E. P. Merkes, “On products of starlike functions," Proceedings of the American Mathematical Society, vol. 13, pp. 479-492, 2013.

[6] M. Nunokawa, "On the multivalent functions," Indian Journal of Pure and Applied Mathematics, vol. 20, no. 6, pp. 577-582, 1989.

[7] M. Nunokawa and S. Owa, "On certain subclass of analytic functions," Indian Journal of Pure and Applied Mathematics, vol. 19, no. 1, pp. 51-54, 1988.

[8] J. Dziok, "Applications of the Jack lemma," Acta Mathematica Hungarica, vol. 105, no. 1-2, pp. 93-102, 2004.

[9] A. E. Livingston, " $p$-valent close-to-convex functions," Soochow Journal of Mathematics, vol. 21, pp. 161-179, 1995.

[10] D. A. Patil and N. K. Thakare, "On convex hulls and extreme points of $p$-valent starlike and convex classws with applications," Bulletin Mathématique de la Société des Sciences Mathématiques de la République Socialiste de Roumanie, vol. 27, no. 75, pp. 145$160,1983$.
[11] S. Owa, "On certain classes of $p$-valent functions with negative coefficients," Simon Stevin A: Quarterly Journal of Pure and Applied Mathematics, vol. 59, no. 4, pp. 385-402, 1985.

[12] R. Bharati, R. Parvatham, and A. Swaminathan, "On subclasses of uniformly convex functions and corresponding class of starlike functions," Tamkang Journal of Mathematics, vol. 28, no. 1, pp. 17-32, 1997.

[13] S. Kanas and A. Wisniowska, "Conic regions and $k$-uniform convexity," Journal of Computational and Applied Mathematics, vol. 105, no. 1-2, pp. 327-336, 1999.

[14] A. W. Goodman, "On uniformly starlike functions," Journal of Mathematical Analysis and Applications, vol. 155, pp. 364-370, 1991.

[15] A. W. Goodman, "On uniformly convex functions," Polska Akademia Nauk: Annales Polonici Mathematici, vol. 56, no. 1, pp. 87-92, 1991.

[16] J. W. Alexander, "Functions which map the interior of the circle upon simple regions," Annals of Mathematics, vol. 14, pp. 12-22, 1915.

[17] Y. J. Kim and E. P. Merkes, "On an integral of powers of a spirallike function," Kyungpook Mathematical Journal, vol. 12, pp. 249-252, 1972.

[18] J. A. Pfaltzgraff, "Univalence of the integral of $f^{\prime}(z)^{\lambda}$," The Bulletin of the London Mathematical Society, vol. 7, no. 3, pp. 254-256, 1975.

[19] D. Breaz and L. Stanciu, "Some properties of a general integral operator," Bulletin of the Transilvania University of Brasov Series III: Mathematics, Informatics, Physics, vol. 5, no. 54, pp. 67-72, 2012.

[20] D. Breaz and N. Breaz, “Two integral operator," Studia Universitatis Babes-Bolyai, vol. 47, no. 3, pp. 13-19, 2002.

[21] D. Breaz, S. Owa, and N. Breaz, "A new integral univalent operator," Acta Universitatis Apulensis. Mathematics-Informatics, no. 16, pp. 11-16, 2008.

[22] E. Deniz, H. Orhan, and H. M. Srivastava, "Some sufficient conditions for univalence of certain families of integral operators involving generalized Bessel functions," Taiwanese Journal of Mathematics, vol. 15, no. 2, pp. 883-917, 2011.

[23] E. Deniz, "Convexity of integral operators involving generalized Bessel functions," Integral Transforms and Special Functions, vol. 24, no. 3, pp. 201-216, 2013.

[24] B. A. Frasin, "Sufficient conditions for integral operator defined by Bessel functions," Journal of Mathematical Inequalities, vol. 4, no. 2, pp. 301-306, 2010.

[25] V. Pescar, "The univalence and the convexity properties for a new integral operator," Studia Universitatis Babes-Bolyai, vol. 56, no. 4, pp. 65-69, 2011.

[26] D. Breaz, "A convexity property for an integral operator on the class $\mathcal{S}_{p}(\beta)$," Journal of Inequalities and Applications, vol. 2008, Article ID 143869, 4 pages, 2008.

[27] B. A. Frasin, "Some sufficient conditions for certain integral operator," Journal of Inequalities and Applications, vol. 2, no. 4, pp. 527-535, 2008.

[28] S. Bulut, "A note on the paper of Breaz and Guney," Journal of Mathematical Inequalities, vol. 2, no. 4, pp. 549-553, 2008.

[29] D. Breaz and V. Pescar, "Univalence conditions for some general integral operators," Banach Journal of Mathematical Analysis, vol. 2, no. 1, pp. 53-58, 2008.

[30] D. Breaz and H. O. Guney, "The integral operator on the classes $\mathcal{S}_{\alpha}^{*}(b)$ and $\mathscr{C}_{\alpha}(b)$, , Journal of Mathematical Inequalities, vol. 2, no. 1, pp. 97-100, 2008. 
[31] S. Bulut, "Convexity properties of a new general integral operator of $p$-valent functions," Mathematical Journal of Okayama University, vol. 56, pp. 171-178, 2014.

[32] B. A. Frasin, "New general integral operators of $p$-valent functions," Journal of Inequalities in Pure and Applied Mathematics, vol. 10, no. 4, article 109, 2009.

[33] B. A. Frasin, "Convexity of integral operators of $p$-valent functions," Mathematical and Computer Modelling, vol. 51, no. 5-6, pp. 601-605, 2010.

[34] A. Mohammed, M. Darus, and D. Breaz, "New criterion for starlike integral operators," Analysis in Theory and Applications, vol. 29, no. 1, pp. 21-26, 2013.

[35] G. S. Salagean, "Subclasses of univalent functions," in Complex Analysis, vol. 1013 of Lecture Notes in Mathematics, pp. 362-372, Springer, Berlin, Germany, 1983.

[36] S. Ruscheweyh, "New criteria for univalent functions," Proceedings of the American Mathematical Society, vol. 49, no. 1, pp. 109$115,1975$.

[37] F. M. Al-Oboudi, "On univalent functions defined by a generalized Sălăgean operator," International Journal of Mathematics and Mathematical Sciences, vol. 2004, no. 27, pp. 1429-1436, 2004.

[38] B. C. Carlson and D. B. Shaffer, "Starlike and prestarlike hypergeometric functions," SIAM Journal on Mathematical Analysis, vol. 15 , no. 4, pp. 737-745, 1984.

[39] B. A. Frasin, "General integral operator defined by Hadamard product," Matematichki Vesnik, vol. 62, no. 2, pp. 127-136, 2010.

[40] D. Breaz, H. Guney, and G. Salagean, "A new integral operator," in Proceedings of the 7th Joint Conference on Mathematics and Computer Science, Cluj, Romania, July 2008.

[41] C. Selvaraj and K. R. Karthikeyan, "Sufficient conditions for univalence of a general integral operator," Bulletin of the Korean Mathematical Society, vol. 46, no. 2, pp. 367-372, 2009.

[42] G. I. Oros and D. Breaz, "Sufficient conditions for univalence of an integral operator," Journal of Inequalities and Applications, vol. 2008, Article ID 127645, 7 pages, 2008.

[43] S. Bulut, "Sufficient conditions for univalence of an integral operator defined by Al-Oboudi differential operator," Journal of Inequalities and Applications, vol. 2008, Article ID 957042, 5 pages, 2008.

[44] S. Bulut, "Some properties for an integral operator defined by Al-Oboudi differential operator," Journal of Inequalities in Pure and Applied Mathematics, vol. 9, no. 4, article 115, 2008.

[45] R. M. El-Ashwah, M. K. Aouf, A. Shamandy, and S. M. El-Deeb, "Properties of certain classes of $p$-valent functions associated with new integral operators," The American Journal of Pure and Applied Mathematics, vol. 2, pp. 79-80, 2013.

[46] G. Saltik Ayhanoz and E. Kadioglu, "Some results of p-valent functions defined by integral operators," Acta Universitatis Apulensis. Mathematics. Informatics, no. 32, pp. 69-85, 2012.

[47] G. Saltik, E. Deniz, and E. Kadioglu, “Two new general p-valent integral operators," Mathematical and Computer Modelling, vol. 52, no. 9-10, pp. 1605-1609, 2010.

[48] D. Breaz and N. Breaz, "Some convexity properties for a general integral operator," Journal of Inequalities in Pure and Applied Mathematics, vol. 7, no. 5, article 177, 8 pages, 2006.

[49] A. Mohammed, M. Darus, and D. Breaz, "Some properties for certain integral operators," Acta Universitatis ApulensisMatematics-Informatics, no. 23, pp. 79-89, 2010.

[50] E. Deniz, M. Caglar, and H. Orhan, "Some convexity properties for two new p-valent integral operators," Hacettepe Journal of Mathematics and Statistics, vol. 40, no. 6, pp. 829-837, 2011. 


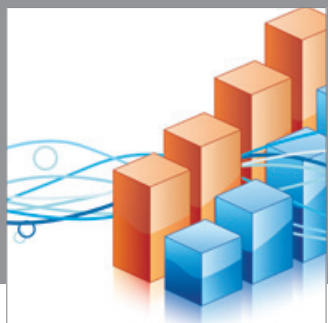

Advances in

Operations Research

mansans

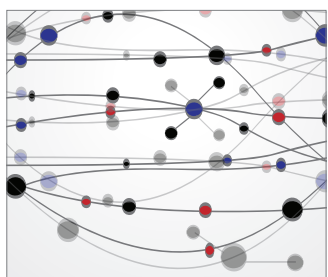

The Scientific World Journal
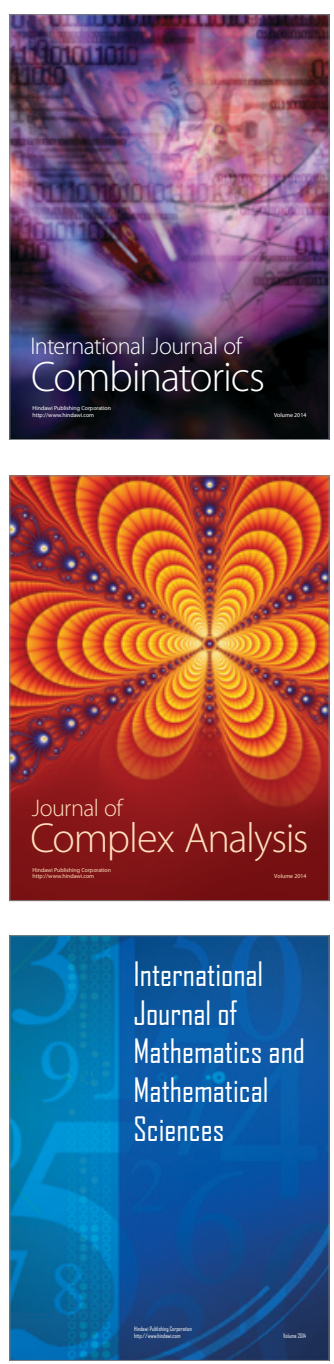
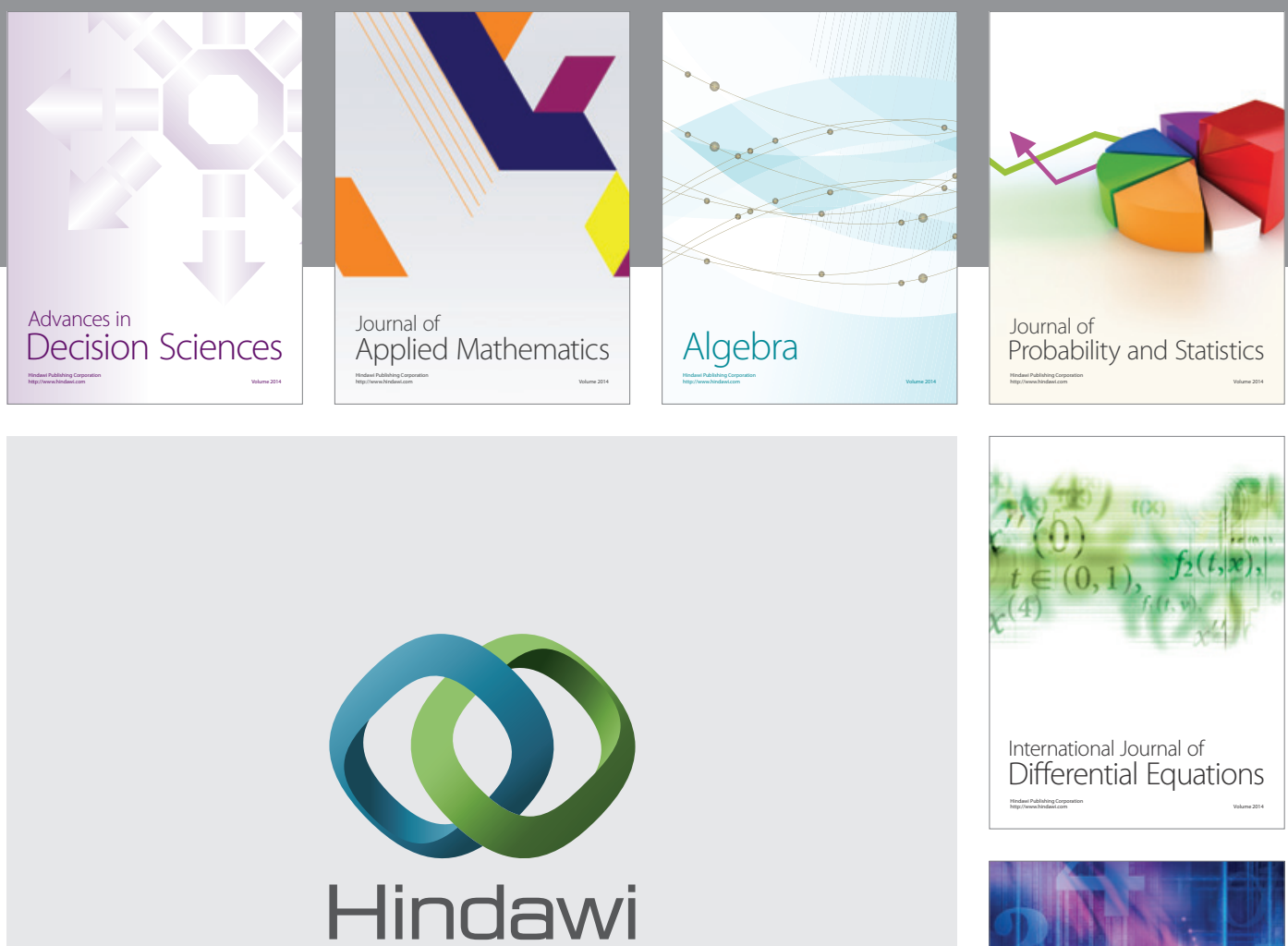

Submit your manuscripts at http://www.hindawi.com
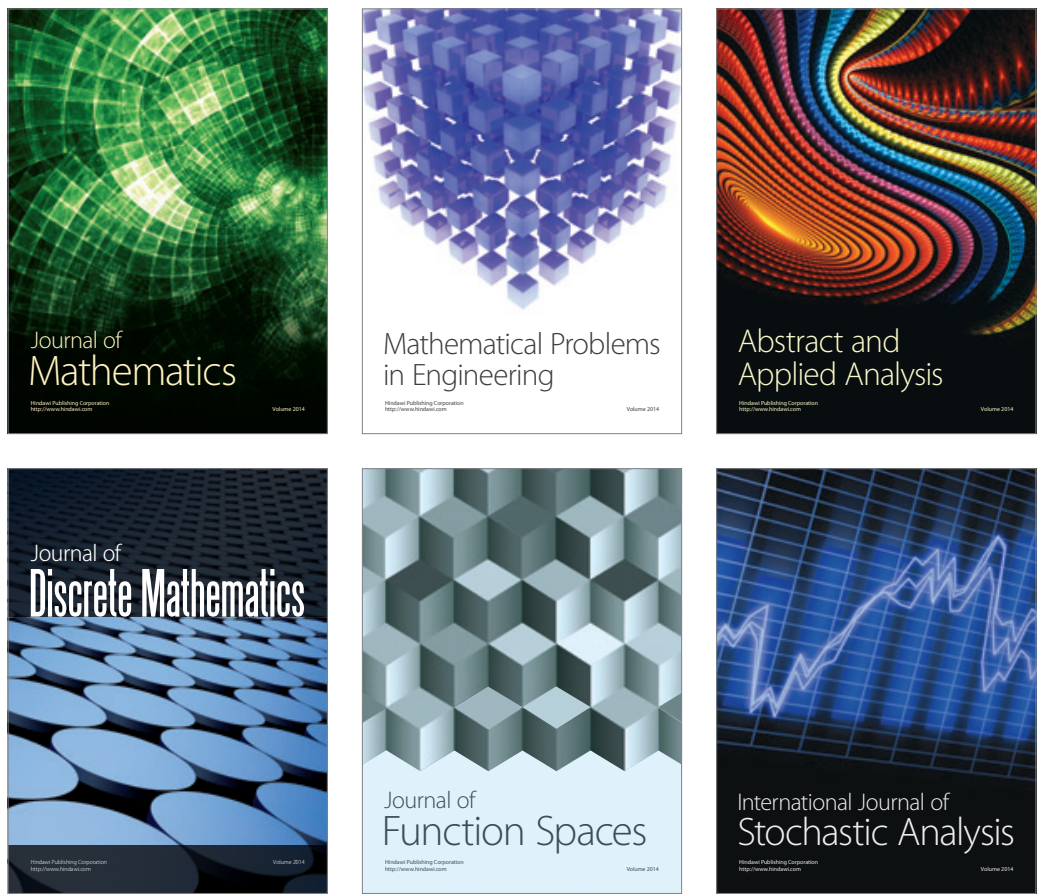

Journal of

Function Spaces

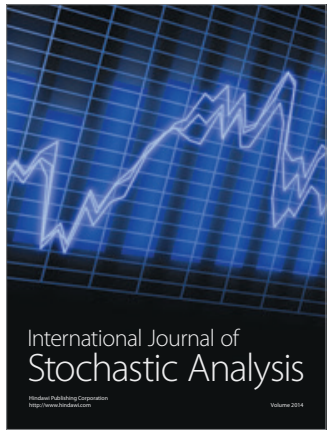

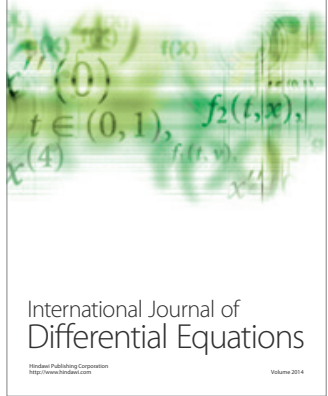
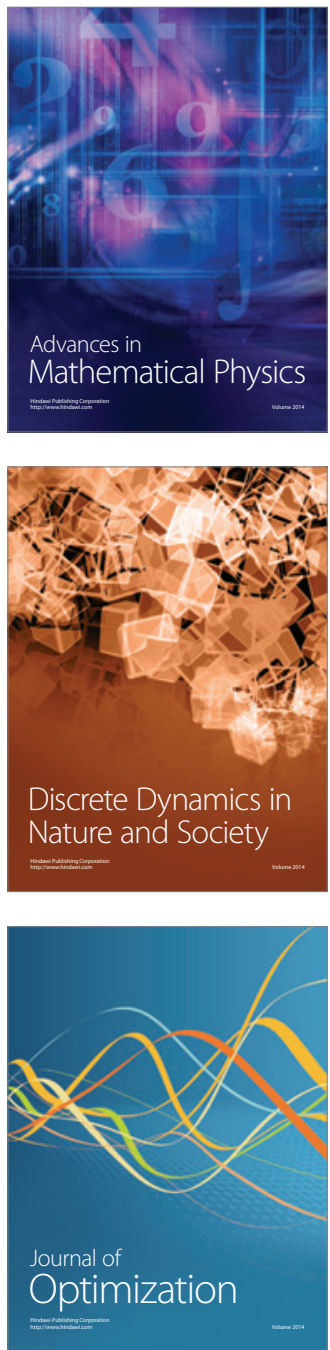